\title{
Atrial-selective sodium channel block by dronedarone: sufficient to terminate atrial fibrillation?
}

\author{
Joachim R. Ehrlich • Dobromir Dobrev
}

Received: 18 February 2011 /Accepted: 23 February 2011 / Published online: 4 May 2011

(C) Springer-Verlag 2011

Presently available drugs used for atrial fibrillation (AF) therapy have major limitations (moderate efficacy and increased risk of potentially life-threatening proarrhythmia), making new drug developments crucial. Novel drugs like the multichannel blocker dronedarone have the potential to convert AF to prevent AF-related stroke and to reduce cardiovascular morbidity and mortality, opening new directions for innovative antiarrhythmic options. Here we summarize mechanistic evidence for atrial-selective sodium channel block as anti-AF principle putting it into perspective to the work by Bogdan et al. 2011, which demonstrated stronger sodium current inhibition by dronedarone at depolarized potentials resulting in atrial selectivity. We also discuss pertinent facts about remodeling processes induced by and leading to AF which likely explain why pharmacological cardioversion is effective in very early stages of AF only.

Atrial selectivity of sodium channel block is a novel antiAF approach that exploits atrial-ventricular differences in sodium current properties (more negative half-inactivation in atria) and in resting membrane potential (more positive in atria leaving a greater proportion of sodium channels in the inactivated state), leading to reduced atrial current availability

J. R. Ehrlich ( $)$

Division of Cardiology, Section of Electrophysiology,

J.W. Goethe-University,

Theodor Stern Kai 7,

60590 Frankfurt, Germany

e-mail: j.ehrlich@em.uni-frankfurt.de

D. Dobrev $(\bowtie)$

Division of Experimental Cardiology, Medical Faculty

Mannheim, Ruprecht-Karls-University Heidelberg,

Theodor-Kutzer-Ufer 1-3,

68167 Mannheim, Germany

e-mail: dobromir.dobrev@medma.uni-heidelberg.de at any given membrane potential. Therefore inactivated-state sodium channel blockers like dronedarone preferentially suppress atrial sodium currents, especially during rapid atrial rhythms like in AF. However, dronedarone less effectively terminates AF compared to amiodarone having only a moderate overall efficacy. Much more work is needed to precisely define the contribution of atrial-selective sodium current inhibition for dronedarone's overall anti-AF efficacy.

$\mathrm{AF}$ is an increasing health problem and is associated with substantial cardiovascular morbidity and mortality, with stroke representing the most critical complication. Presently available drugs used for therapy of AF (antiarrhythmic drugs and anticoagulants) have major limitations because of moderate efficacy and inherent risk of potentially life-threatening proarrhythmia or bleeding complications, making new drug developments crucial (Dobrev and Nattel 2010). Novel drugs like dronedarone have the potential to prevent AF-related stroke and to reduce cardiovascular morbidity and mortality (Hohnloser et al. 2009), opening a new avenue for innovative antiarrhythmic options.

In January 2010 dronedarone has been approved for the treatment of nonpersistent AF in Europe. It is an antiarrhythmic compound with rate- and rhythm-controlling properties, which has been developed as a structurally related, multichannelblocking congener of amiodarone with a similarly broad spectrum of inhibitory actions on ion channels (Ehrlich and Nattel 2009). In the April issue of Naunyn-Schmiedeberg's Archive of Pharmacology, Bogdan and colleagues (2011) illustrate the mode of dronedarone action on sodium channels and identify a peculiar behavior of the substance to inhibit inactivated channels at more depolarized potentials more effectively, leading to atrial-selective sodium channel block. They demonstrate a similar behavior for calcium channels and find inhibitory action on hyperpolarization-activated currents 
(HCN4), which are critically involved in physiological sinus node automaticity.

Atrial-selective inhibition of sodium channels has previously been demonstrated for several antiarrhythmic agents (Burashnikov et al. 2007; Sicouri et al. 2010) and represents a property likely contributing to dronedarone's antiarrhythmic effect. Atrial sodium channel blockade leads to alterations of various biophysical parameters of sodium current $\left(\mathrm{I}_{\mathrm{Na}}\right)$ that produce post-repolarization refractoriness without a major effect on action potential duration (APD). Post-repolarization refractoriness is defined as difference between effective refractory period (ERP) and $\mathrm{APD}_{75}$ in atria and difference between ERP and $\mathrm{APD}_{90}$ in ventricles. Ion channel inhibition is the conventional and still the most commonly applied principle for pharmacological treatment of cardiac arrhythmias. However, once the arrhythmia has occurred, AF by itself alters expression profile and biophysical behavior of cardiac ion channels (atrial electrical remodeling, see below), further enhancing the severity of the substrate and increasing the likelihood of AF to sustain (Nattel et al. 2008). The precise signaling that creates the arrhythmogenic substrate is incompletely understood, but is shown to involve $\mathrm{Ca}^{2+} /$ calmodulin/calcineurin-dependent pathways (Dobrev and Nattel 2010). Likely because of the presence of such remodeling and the lack of an effect on related signaling, most antiarrhythmic drugs do neither arrest arrhythmia progression nor improve clinical outcome. Dronedarone is the first antiarrhythmic compound with clinically proven beneficial effect on morbidity and mortality in patients with AF (Hohnloser et al. 2009). It reduces AF recurrences after cardioversion and has low risk of relevant side effects. Owing to these issues, the recently published European guidelines for $\mathrm{AF}$ management have suggested dronedarone as first-line drug option for patients with nonpersistent $\mathrm{AF}$ (Camm et al. 2010). Despite the generally favorable safety profile, two recent cases of significant liver toxicity have been reported, which is not surprising based on structural similarities between dronedarone and amiodarone. Such significant toxicity would represent a great shortcoming to dronedarone if found to be causally related to the substance (http://www.fda.gov/Drugs/DrugSafety/ucm240011.htm). Prospectively collected data in larger patients populations are clearly needed to further evaluate the safety profile of dronedarone in the different patient populations.

\section{AF-related atrial electrical remodeling: why does it matter?}

AF most commonly occurs in hearts that have been affected by and are modified through remodeling processes due to clinical conditions such as hypertension, coronary artery disease, or heart failure. Each disease entity causes specific atrial changes that contribute to development of diverse arrhythmia substrates (Schotten et al. 2011). Hypertension for instance increases atrial pressure and may lead to enhanced atrial fibrosis through angiotensin-mediated pathways with subsequently enhanced vulnerability to $\mathrm{AF}$ (Burstein and Nattel 2008). Heart failure is associated with a high prevalence of AF and leads to specific remodeling of the atria by promoting atrial fibrosis and impaired impulse conduction that maintain the arrhythmia (Ehrlich et al. 2002). Coronary artery disease may similarly enhance regional heterogeneity of impulse conduction in conjunction with enhanced automaticity (Nishida et al. 2011).

Mechanistically, AF is constituted by multiple reentrant or spiral waves (rotors) that lead to rapid and repetitive activation of the atria. The AF-associated electrical remodeling is characterized by shortened atrial APD, abbreviated atrial ERP with increased impulse conduction heterogeneity and conduction slowing that increase propensity to reentrant activity (Nattel et al. 2008). Wijffels et al. documented this self-perpetuating nature of $\mathrm{AF}$ in classical animal experiments through continuous re-induction of the arrhythmia via implanted pacemakers over a period of weeks (Wijffels et al. 1995). A similar behavior can be observed in many patients where AF progresses from the paroxysmal form to persistent AF (Jahangir et al. 2007), perhaps as a consequence of remodeling.

Several mechanisms contribute to AF persistence like ectopic activity (e.g., focal activation from specific sites such as great cardiac veins) or circles of macro-reentry, but the final common pathway of these processes is multiple wavelet reentry (Nattel et al. 2008). Maintenance of reentry critically depends on the presence of short wavelengths that can mathematically be represented as the product of conduction velocity times refractory period. If a cardiac disease reduces speed of impulse conduction by changing $\mathrm{Na}^{+}$channel availability, increasing the amount of atrial fibrosis or altering intercellular communication through connexins, the wavelength is shortened and a substrate for reentry is created (Dhein et al. 2010). Similarly, if shortening of the ERP occurs by decreasing APD, the likelihood of reentry is increased (Dobrev 2006; Nattel et al. 2008). Enhanced anisotropic conduction and heterogeneity of repolarization are further associated with important disturbances in impulse propagation that may also lead to reentrant arrhythmias (Dhein et al. 2010). Consistent with these concepts, human APD is shortened with impaired rate adaptation during AF (Nattel et al. 2008). Atrial APD, and thus ERP, is determined by the balance between inward currents that depolarize and outward currents that repolarize atrial myocytes. Studies in AF patients showed that decreased L-type $\mathrm{Ca}^{2+}$ current $\left(\mathrm{I}_{\mathrm{Ca}, \mathrm{L}}\right.$; reduced depolarization power), increased inward rectifier $\mathrm{K}^{+}$current $\mathrm{I}_{\mathrm{K} 1}$, and the constitutively active acetylcholine-dependent $\mathrm{K}^{+}$current $\mathrm{I}_{\mathrm{K}}$, $\mathrm{ACh}$ (enhanced repolarization power) are major contributors 
to APD shortening (Dobrev et al. 2005; Girmatsion et al. 2009; van Wagoner et al. 1999). In addition to fibrosis, slightly reduced peak sodium current $\left(\mathrm{I}_{\mathrm{Na}}\right)$ amplitude but enhanced late sodium current, $\mathrm{I}_{\mathrm{Na} \text {,late }}$ (Sossalla et al. 2010), and decreased connexin-40 expression (Gaborit et al. 2005) add to atrial conduction disturbances.

These AF-related changes in ion-channel properties alter the response to antiarrhythmic drugs, principally making $\mathrm{AF}$ more drug resistant. A poorer response of persistent AF has been shown for both $\mathrm{Na}^{+}-$and $\mathrm{K}^{+}$-channel blockers (Duytschaever et al. 2005). Accordingly, ion-channel remodeling by itself represents a potential antiarrhythmic drug target. Amiodarone treatment suppresses APD abbreviation during experimental AF (Shinagawa et al. 2003), whereas $\mathrm{Ca}^{2+}-, \mathrm{K}^{+}$-, and $\mathrm{Na}^{+}$-channel blockers are ineffective in that respect. Therefore, it has been assumed that prevention of ion-channel remodeling (suppression of $\mathrm{I}_{\mathrm{Ca}, \mathrm{L}}$ reduction) may contribute to amiodarone's superior efficacy in AF (Shinagawa et al. 2003). Inhibition of two-poredomain TASK-1 leak channels, which are preferentially expressed in atria, may also contribute to amiodarone's antiAF efficacy (Gierten et al. 2010). Whether suppression of $\mathrm{I}_{\mathrm{Ca}, \mathrm{L}}$ remodeling and inhibition of TASK-1 channels contribute to the antiarrhythmic effects of dronedarone is unknown.

\section{Paradoxon of $\mathbf{I}_{\mathrm{Na}}$ inhibition to terminate $\mathrm{AF}$}

The efficacy of sodium channel-blocking drugs for termination of $\mathrm{AF}$ is difficult to explain in the context of the classical multiple wavelet hypothesis outlined above. Such pharmacological agents should theoretically promote $\mathrm{AF}$ maintenance as they decrease conduction velocity by reducing $\mathrm{I}_{\mathrm{Na}}$ and shortening wavelength. Paradoxically, and contrary to these predictions, many class I substances like flecainide and propafenone are effective in converting a large proportion of recent-onset AF (Capucci et al. 1994; Suttorp et al. 1989). These drugs are nonselective sodium channel blockers and additionally target other ion channels (Hwang et al. 2011; Voigt et al. 2010) involved in the remodeling process (Dobrev et al. 2005; Dobrev and Nattel 2010). This paradoxon might possibly be understood if we consider alternative concepts of functional reentry. According to spiral wave (rotor) theory (and in contrast to classical reentry concepts), atrial excitation during AF is viewed as spiral waves with rotors that meander through the atria with a gradient of excitability that makes activation in the center of a rotor slower than in the periphery (Jalife et al. 2002). Once atrial excitability is reduced by sodium channel blockade, the rotor frequency decreases because the radius of the spiral wave increases, thereby preventing rotor stabilization and enhancing the likelihood of AF termina- tion (Kneller et al. 2001). Although this theory is appealing, the precise mechanism of $\mathrm{AF}$ termination by sodium channel block remains unclear. The higher efficacy of class I antiarrhythmic drugs like vernakalant in cardioverting recent-onset compared with persistent AF (Ehrlich and Nattel 2009) might result from the generally lower degree of atrial remodeling in recent-onset AF. Due to the more complex pathophysiology in persistent $\mathrm{AF}$ with progressive structural changes in both atria (structural remodeling), AF stabilization in such patients may depend on structural rather than ion-channel remodeling. Complex interaction patterns between myocytes and fibroblasts may lead to reentrant circuits that depend to a lesser extent on functional reentry determinants like properties of $\mathrm{I}_{\mathrm{Na}}$ currents.

\section{Is atrial-selective $\mathbf{I}_{\mathrm{Na}}$ inhibition sufficient to terminate AF?}

As mentioned above class I antiarrhythmic agents like flecainide and propafenone are effective and commonly used agents for pharmacological AF cardioversion and prevention of $\mathrm{AF}$ recurrence in patients with no or lowdegree structural heart disease. However, since they may precipitate deleterious side effects in individuals with coronary artery disease, their clinical applicability is limited (Camm et al. 2010).

Recent evidence from animal models of AF documented greater atrial vs ventricular $\mathrm{I}_{\mathrm{Na}}$ current densities and atrial sodium channel inactivation at more negative potentials (Burashnikov et al. 2007). Since atrial cardiomyocytes have a more depolarized resting membrane potential, the atrial diastolic take-off potential for excitation is further elevated at faster rates (like during $\mathrm{AF}$ ), which additionally accentuates preset differences in resting membrane potential. This peculiar behavior effectively differentiates ventricular from atrial APs constituting a potential target for atrium-selective anti-AF therapy (Fig. 1). Ranolazine, which is a predominant open-channel $\mathrm{I}_{\mathrm{Na}}$ blocker with inhibitory effect on $\mathrm{I}_{\mathrm{Na} \text {,late, }}$, produced use-dependent depression of sodium channel parameters, as indirectly evidenced by a decreased rate of rise of action potential upstroke, conduction velocity and diastolic threshold of excitation (Burashnikov et al. 2007), thereby inducing antiarrhythmic post-repolarization refractoriness. Chronic amiodarone treatment exerted similar atrial-selective effects on cardiac $\mathrm{I}_{\mathrm{Na}}$. Under experimental conditions-particularly at faster rates - amiodarone and dronedarone, both of which are inactivated-state sodium channel blockers with rapid dissociation kinetics, have a greater potential to depress $\mathrm{I}_{\mathrm{Na}}$ properties in atria than in ventricles (Burashnikov and Antzelevitch 2010). Recently, a combination of ranolazine 

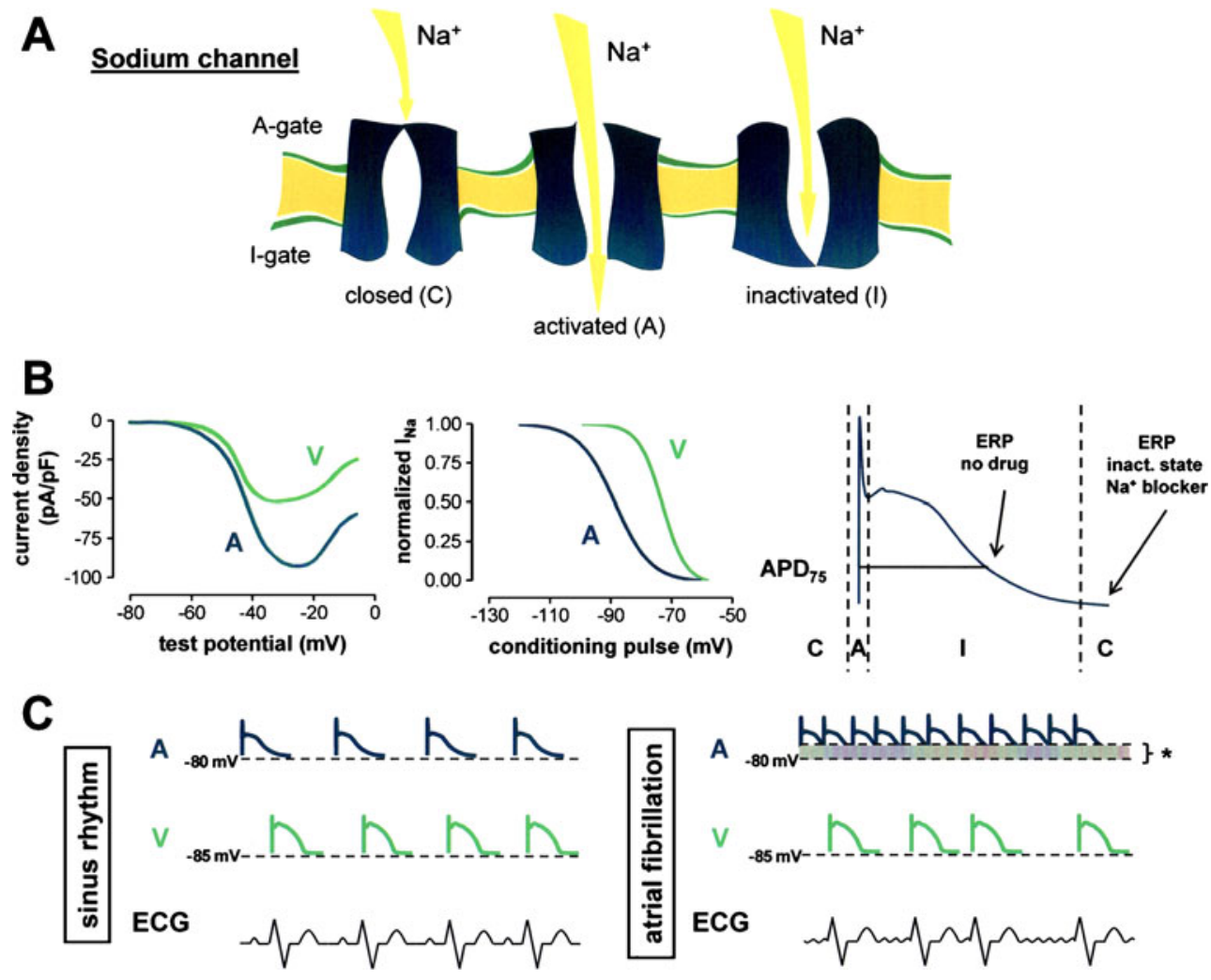

Fig. 1 Atrial vs ventricular sodium current differences as determinants of atrial-selective actions of antiarrhythmic drugs. a A schematic representation of activated, inactivated, and closed states of sodium channels at the membrane. For simplification only the pore area is illustrated. Sodium ion entry into the cell occurs if both gates (A- and I-gate) are open. b Left, simplified characteristics of atrial $(A)$ vs ventricular $(V)$ sodium current characteristics as identified by Burashnikov et al. (2007). Atrial currents are larger, peak at more positive potentials than ventricular currents. Atrial sodium current inactivation occurs already at more negative potentials, leading to greater channel unavailability at any given membrane potential. Right, a schematic of an atrial action potential (AP) is illustrated. The dotted lines illustrate sodium channel states during a given period of the AP ( $C$ closed, $A$ activated [open], $I$ inactivated). AP duration $(A P D)$ at

and dronedarone (two mechanistically different sodium channel blockers with additive effects on $\mathrm{I}_{\mathrm{Na}}$ properties, see above) were highly effective for AF termination and prevention and found the combination highly effective (Sicouri et al. 2010). As hypothesized, both substances synergistically potentiated their antiarrhythmic efficacies, with the combination being much more effective than expected from the mathematical sum of the two separate efficacy values. Interestingly, pulmonary vein cardiomyocytes, which accommodate important triggers for clinical AF episodes, have more depolarized resting membrane potential than left atrial cardiomyocytes (Ehrlich et al. 2003) and may accordingly be even more prone to atrialselective sodium channel block. This may provide an additional explanation why patients with recent-onset or short-term paroxysmal $\mathrm{AF}$, in which $\mathrm{AF}$ mainly comes from

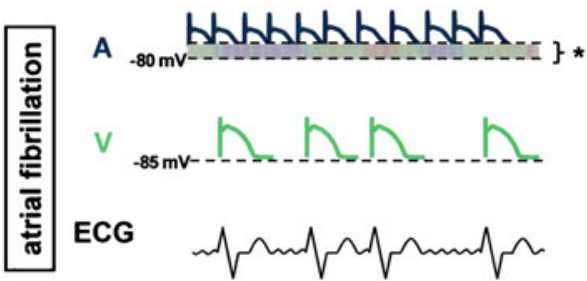

$75 \%$ repolarization typically coincides with effective refractory period $(E R P)$. If an inactivated-state sodium channel blocker is added, ERP will be prolonged beyond repolarization, leading to post-repolarization refractoriness. c Atrial $(A)$ and ventricular $(V)$ APs are schematically illustrated during sinus rhythm and atrial fibrillation. During more rapid atrial activation like in $\mathrm{AF}$, the diastolic take-off potential is further elevated (asterisk), increasing sodium channel inactivation and the efficacy of inactivated-state sodium channel blocker like dronedarone. For the purpose of simplification, the role of rapid vs slow unbinding characteristics of sodium channel blockers has not been illustrated in this figure. A-gate activation gate, I-gate inactivation gate. b and c contain data modified from Burashnikov et al. (2007) with permission

the pulmonary veins region, show a higher AF cardioversion rate with sodium channel block than patients with persistent $\mathrm{AF}$, in which $\mathrm{AF}$ rarely depends on electrical activity from pulmonary veins.

The paper by Bogdan et al. presents results of a combined in vivo and in vitro study regarding the effects of dronedarone on monophasic APs, peak $\mathrm{I}_{\mathrm{Na}}$, and $\mathrm{I}_{\mathrm{Ca}, \mathrm{L}}$ as well as heterologously expressed HCN4 channels (Bogdan et al. 2011). Through changes in holding potential, they documented a strong potential-dependent efficacy of $\mathrm{I}_{\mathrm{Na}}$ blockade by dronedarone. For instance at $-80 \mathrm{mV}$, dronedarone halfmaximally inhibited $\mathrm{I}_{\mathrm{Na}}$ with an $\mathrm{IC}_{50}$ of $\sim 0.7 \mu \mathrm{mol} / \mathrm{L}$, while at $-100 \mathrm{mV}$ the substance was largely ineffective, with $10 \mu \mathrm{mol} / \mathrm{L}$ producing only $\sim 9 \%$ current inhibition. The authors extend these findings on $\mathrm{I}_{\mathrm{Na}}$ to a similar observation with $\mathrm{I}_{\mathrm{Ca}, \mathrm{L}}$. Here they calculated an $\mathrm{IC}_{50}$ of $\sim 0.4 \mu \mathrm{mol} / \mathrm{L}$ 
at $-40 \mathrm{mV}$, while at $-80 \mathrm{mV}$ dronedarone inhibited only a minor portion of the current $(\sim 20 \%)$. These findings are consistent with previously published observations in canines and extend these recent data by quantifying the relative effectiveness of dronedarone at various membrane potentials. Overall, these data point to an atrial-selective action of dronedarone on $\mathrm{I}_{\mathrm{Na}}$ in vivo. However, acute dronedarone is still less effective than acute amiodarone at clinically relevant concentrations to terminate experimental AF (Burashnikov et al. 2010). Together with data on clinical efficacy of the substance to convert AF (Touboul et al. 2003), this suggests that atrial-specific sodium channel inhibition by dronedarone is by itself not sufficient for AF conversion.

It is difficult to directly extrapolate experimental findings obtained in animals or expression systems to human wholeheart physiology. In the present study, the authors utilized measurements of APs in pigs and studied ventricular guineapig cardiomyocytes, adding another potential confounder by comparing different species. It is very important to note that composition and characteristics of ion channels may differ substantially between species and between cardiac regions. As the authors correctly acknowledge, the molecular composition of atrial sodium channels might not be equal to that of sodium channels in ventricular myocardium. Nevertheless, the results presented in the paper by Bogdan et al. were intrinsically consistent and compatible with previous data suggesting a minor role of species-specific differences in this context (Bogdan et al. 2011).

\section{Conclusions}

In summary, the study of Bogdan et al. 2011 demonstrates atrial-selective $\mathrm{I}_{\mathrm{Na}}$ inhibition by dronedarone that is likely to contribute to the substance's anti-AF efficacy and its limited proarrhythmic effects at the ventricular level. Further extensive work is needed to explore other mechanisms underlying the therapeutic value of dronedarone in $\mathrm{AF}$ and to more precisely define the contribution of atrialselective $\mathrm{I}_{\mathrm{Na}}$ inhibition for the drug's overall anti-AF efficacy.

\begin{abstract}
Acknowledgments The help of Moritz Toenne, BSc with artwork is gratefully acknowledged. Dr. Ehrlich received funding from the Deutsche Herzstiftung and Else-Kröner-Fresenius Stiftung. Dr. Dobrev's research is supported by the German Federal Ministry of Education and Research Atrial Fibrillation Competence Network (grant 01Gi0204), the Deutsche Forschungsgemeinschaft (Do 769/ 1-3), the European Union (European Network for Translational Research in Atrial Fibrillation, EUTRAF), and the Foundation Leducq (European-North American Atrial Fibrillation Research Alliance, ENAFRA).
\end{abstract}

Conflict of interest Dr. Ehrlich has received lecture fees from Sanofi-Aventis. Dr. Dobrev reports no conflict of interest.

\section{References}

Bogdan R, Goegelein H, Ruetten H (2011) Effect of dronedarone on $\mathrm{Na}+, \mathrm{Ca} 2+$ and $\mathrm{HCN}$ channels. Naunyn Schmiedebergs Arch of Pharmacol 383(4):347-356

Burashnikov A, Antzelevitch C (2010) New developments in atrial antiarrhythmic drug therapy. Nat Rev Cardiol 7(3):139-148

Burashnikov A, Di Diego JM, Zygmunt AC, Belardinelli L, Antzelevitch C (2007) Atrium-selective sodium channel block as a strategy for suppression of atrial fibrillation: differences in sodium channel inactivation between atria and ventricles and the role of ranolazine. Circulation 116(13):1449-1457

Burashnikov A, Belardinelli L, Antzelevitch C (2010) Acute dronedarone is inferior to amiodarone in terminating and preventing atrial fibrillation in canine atria. Heart Rhythm 7(9):1273-1279

Burstein B, Nattel S (2008) Atrial fibrosis: mechanisms and clinical relevance in atrial fibrillation. J Am Coll Cardiol 51(8):802-809

Camm AJ, Kirchhof P, Lip GY, Schotten U, Savelieva I, Ernst S, Van Gelder IC, Al AN, Hindricks G, Prendergast B, Heidbuchel H, Alfieri O, Angelini A, Atar D, Colonna P, De Caterina R, De Sutter J, Goette A, Gorenek B, Heldal M, Hohloser SH, Kolh P, Le Heuzey JY, Ponikowski P, Rutten FH (2010) Guidelines for the management of atrial fibrillation: the Task Force for the Management of Atrial Fibrillation of the European Society of Cardiology (ESC). Eur Heart J 31(19):2369-2429

Capucci A, Boriani G, Botto GL, Lenzi T, Rubino I, Falcone C, Trisolino G, Della CS, Binetti N, Cavazza M (1994) Conversion of recent-onset atrial fibrillation by a single oral loading dose of propafenone or flecainide. Am J Cardiol 74(5):503-505

Dhein S, Hagen A, Jozwiak J, Dietze A, Garbade J, Barten M, Kostelka M, Mohr FW (2010) Improving cardiac gap junction communication as a new antiarrhythmic mechanism: the action of antiarrhythmic peptides. Naunyn Schmiedebergs Arch Pharmacol 381(3):221-234

Dobrev D (2006) Electrical remodeling in atrial fibrillation. Herz 31 (2):108-112

Dobrev D, Nattel S (2010) New antiarrhythmic drugs for treatment of atrial fibrillation. Lancet 375(9721):1212-1223

Dobrev D, Friedrich A, Voigt N, Jost N, Wettwer E, Christ T, Knaut M, Ravens U (2005) The G protein-gated potassium current I(K, ACh) is constitutively active in patients with chronic atrial fibrillation. Circulation 112(24):3697-3706

Duytschaever M, Blaauw Y, Allessie M (2005) Consequences of atrial electrical remodeling for the anti-arrhythmic action of class IC and class III drugs. Cardiovasc Res 67(1):69-76

Ehrlich JR, Nattel S (2009) Novel approaches for pharmacological management of atrial fibrillation. Drugs 69(7):757-774

Ehrlich JR, Nattel S, Hohnloser SH (2002) Atrial fibrillation and congestive heart failure: specific considerations at the intersection of two common and important cardiac disease sets. J Cardiovasc Electrophysiol 13(4):399-405

Ehrlich JR, Cha TJ, Zhang L, Chartier D, Melnyk P, Hohnloser SH, Nattel S (2003) Cellular electrophysiology of canine pulmonary vein cardiomyocytes: action potential and ionic current properties. J Physiol 551(Pt 3):801-813

Gaborit N, Steenman M, Lamirault G, Le Meur N, Le Bouter S, Lande G, Leger J, Charpentier F, Christ T, Dobrev D, Escande D, Nattel S, Demolombe S (2005) Human atrial ion channel and transporter subunit gene-expression remodeling associated with valvular heart disease and atrial fibrillation. Circulation 112(4):471-481

Gierten J, Ficker E, Bloehs R, Schweizer PA, Zitron E, Scholz E, Karle C, Katus HA, Thomas D (2010) The human cardiac K2P3.1 (TASK-1) potassium leak channel is a molecular target for the class III antiarrhythmic drug amiodarone. Naunyn Schmiedebergs Arch Pharmacol 381(3):261-270 
Girmatsion Z, Biliczki P, Bonauer A, Wimmer-Greinecker G, Scherer M, Moritz A, Bukowska A, Goette A, Nattel S, Hohnloser SH, Ehrlich JR (2009) Changes in microRNA-1 expression and IK1 up-regulation in human atrial fibrillation. Heart Rhythm 6 (12):1802-1809

Hohnloser SH, Crijns HJ, van Eickels M, Gaudin C, Page RL, TorpPedersen C, Connolly SJ (2009) Effect of dronedarone on cardiovascular events in atrial fibrillation. N Engl J Med 360 (7):668-678

Hwang HS, Hasdemir C, Laver D, Mehra D, Turhan K, Faggioni M, Yin H, Knollmann BC (2011) Inhibition of cardiac $\mathrm{Ca} 2+$ release channels (RyR2) determines efficacy of class I antiarrhythmic drugs in catecholaminergic polymorphic ventricular tachycardia. Circ Arrhythm Electrophysiol (in press)

Jahangir A, Lee V, Friedman PA, Trusty JM, Hodge DO, Kopecky SL, Packer DL, Hammill SC, Shen WK, Gersh BJ (2007) Long-term progression and outcomes with aging in patients with lone atrial fibrillation: a 30-year follow-up study. Circulation 115(24):3050-3056

Jalife J, Berenfeld O, Mansour M (2002) Mother rotors and fibrillatory conduction: a mechanism of atrial fibrillation. Cardiovasc Res 54 (2):204-216

Kneller J, Leon J, Nattel S (2001) How do class 1 antiarrhythmic drugs terminate atrial fibrillation? A quantitative analysis based on a realistic ionic model. Circulation 104(17):5-5

Nattel S, Burstein B, Dobrev D (2008) Atrial remodeling and atrial fibrillation: mechanisms and implications. Circ Arrhythm Electrophysiol 1(1):62-73

Nishida K, Qi XY, Wakili R, Comtois P, Chartier D, Harada M, Iwasaki YK, Romeo P, Maguy A, Dobrev D, Michael G, Talajic M, Nattel S (2011) Mechanisms of atrial tachyarrhythmias associated with coronary artery occlusion in a chronic canine model. Circulation 123(2):137-146
Schotten U, Verheule S, Kirchhof P, Goette A (2011) Pathophysiological mechanisms of atrial fibrillation: a translational appraisal. Physiol Rev 91(1):265-325

Shinagawa K, Shiroshita-Takeshita A, Schram G, Nattel S (2003) Effects of antiarrhythmic drugs on fibrillation in the remodeled atrium: insights into the mechanism of the superior efficacy of amiodarone. Circulation 107(10):1440-1446

Sicouri S, Burashnikov A, Belardinelli L, Antzelevitch C (2010) Synergistic electrophysiologic and antiarrhythmic effects of the combination of ranolazine and chronic amiodarone in canine atria. Circ Arrhythm Electrophysiol 3(1):88-95

Sossalla S, Kallmeyer B, Wagner S, Mazur M, Maurer U, Toischer K, Schmitto JD, Seipelt R, Schondube FA, Hasenfuss G, Belardinelli L, Maier LS (2010) Altered $\mathrm{Na}(+)$ currents in atrial fibrillation effects of ranolazine on arrhythmias and contractility in human atrial myocardium. J Am Coll Cardiol 55(21):2330-2342

Suttorp MJ, Kingma JH, Huen LA, Mast EG (1989) Intravenous flecainide versus verapamil for acute conversion of paroxysmal atrial fibrillation or flutter to sinus rhythm. Am J Cardiol 63(11):693-696

Touboul P, Brugada J, Capucci A, Crijns HJ, Edvardsson N, Hohnloser SH (2003) Dronedarone for prevention of atrial fibrillation: a dose-ranging study. Eur Heart J 24(16):1481-1487

van Wagoner DR, Pond AL, Lamorgese M, Rossie SS, McCarthy PM, Nerbonne JM (1999) Atrial L-type Ca currents and human atrial fibrillation. Circ Res 85:428-436

Voigt N, Rozmaritsa N, Trausch A, Zimniak T, Christ T, Wettwer E, Matschke K, Dobrev D, Ravens U (2010) Inhibition of IK,ACh current may contribute to clinical efficacy of class I and class III antiarrhythmic drugs in patients with atrial fibrillation. Naunyn Schmiedebergs Arch Pharmacol 381(3):251-259

Wijffels MC, Kirchhof CJ, Dorland R, Allessie MA (1995) Atrial fibrillation begets atrial fibrillation: a study in awake chronically instrumented goats. Circulation 92:1954-1968 